\title{
As infâncias e a educação infantil em tempos de pandemia: que cenário revelam lives sobre o tema
}

\section{Children and childhood education in pandemic times: what scenario live broadcasts revel on the subject?}

\author{
Las infancias y la educación de la primera infancia en tiempos \\ pandémicos: ¿qué escenario revelan transmisiones de video en vivo \\ acerca del tema?
}

\author{
Natalia Francisca Cardia dos Santos' \\ https://orcid.org/0000-0003-4973-54|4
}

\begin{abstract}
Resumo: Neste registro sistematizamos transmissões de vídeos postadas no Youtube em 2020 a partir do termo chave: educação infantil em tempos de pandemia. Utilizando como metodologia a netnografia, e no recorte de tempo compreendido entre 17 de março e 12 de junho localizamos 78 transmissões, as quais subdividimos em duas categorias, que se tornaram perceptíveis na análise: (i) lives que trazem indicativos de defesa/divulgação (direta ou indireta) de ideias e concepções privadas, e (ii) lives com interesse de análise da conjectura atual, discutindo a educação infantil no contexto da pandemia sob um olhar crítico, apartidário e tendo como premissa principal a defesa das crianças e suas infâncias nesse contexto. Ao sistematizar ações do universo virtual ao registro escrito buscamos garantir que resistam ao tempo e ao risco de que se dissolvam na lógica da velocidade digital, conferindo materialidade a essas tantas análises feitas em tempo real, compondo um acervo documental capaz de dar visibilidade a hiatos existentes ao se pensar ações de atendimento à primeira infância no período de pandemia.
\end{abstract}

Palavras-chave: Educação infantil. Pandemia. Política educacional.

\begin{abstract}
This paper presents an overview of videos that were broadcasted on YouTuve in 2020 on the following subject: early childhood education in pandemic times. Using netnography as methodology, we researched videos that were broadcasted between February 10th and June 12th, and we found 78 broadcasts, which were divided into two categories in order to improve the analysis: (i) live broadcasts that bring indications of defense/dissemination (directly or indirectly) of private ideas and concepts about childhood; and (ii) live broadcasts focused in the analysis of the current conjecture, discussing early childhood education in the pandemic context from a critical, non-partisan perspective and with the main premise of defending children and their childhoods in this context. By systematizing actions from the virtual universe to the written register, we seek to ensure that they resist time, preventing from the risk of dissolving into the logic of the "digital speed", giving
\end{abstract}

\footnotetext{
' Mestre em educação pela Universidade Nove de Julho (UNINOVE), professora de educação básica na rede municipal de ensino de São Paulo, nataliacardia@gmail.com
}

Olhar de professor, Ponta Grossa, v. 24, p. I-18, e-16055.075, 202 I.

Disponível em <https://revistas2.uepg.br/index.php/olhardeprofessor> 
materiality to these analyzes that were done in real time, composing a collection capable of giving visibility to the gaps that exist concerning the assistance for the early childhood in the pandemic period.

Keywords: Early childhood education. Pandemic. Educational politics.

Resumen: En este registro, sistematizamos transmisiones de video publicadas en Youtube en 2020 usando el término clave: educación de la primera infancia en tiempos de pandemia. Utilizando la netnografía como metodología, y en el período comprendido entre el 10 de febrero y el 12 de junio localizamos 78 transmisiones, que subdividimos en dos categorías, que se hicieron notables en el análisis: (i) videos que traen indicios de defensa/difusión (directa o indirecta) de ideas y conceptos privados, y (ii) videos transmitidos con interés en analizar la conjetura actual, discutiendo la educación de la primera infancia en el contexto de la pandemia desde una perspectiva crítica y no partidista y con la premisa principal de defender a los niños y sus infancias en ese contexto. Al sistematizar acciones desde el universo virtual hasta el registro escrito, buscamos garantizar que resistan el tiempo y el riesgo de que se disuelvan en la lógica de la velocidad digital, dando materialidad a estos muchos análisis realizados en tiempo real, componiendo una colección documental capaz de dar visibilidad a las brechas existentes cuando se piensa en la educación de la primera infancia en el período pandémico.

Palabras-clave: Educación de la primera infância. Pandemia. Política educativa.

\section{Introdução}

Neste cenário de pandemia em decorrência do Covid-19 que assola o Brasil desde fevereiro de 2020, especialmente a partir do momento e que foi decretado estado de emergência no país, diversas medidas têm conduzido ao isolamento social da população como forma de conter a propagação da doença, incidindo inclusive na interrupção do funcionamento de escolas e universidades desde então.

E diante desse contexto, nesse período de distanciamento físico que nos foi imposto em virtude da situação, a realização de lives na internet tem sido uma estratégia encontrada por inúmeros grupos com temas e interesses em comum, com vistas a manter vivo o diálogo, a proximidade das interlocuções e as reflexões coletivas.

Por live entenda-se: uma gravação de vídeo transmitida ao vivo na internet, geralmente por meio de plataformas e redes sociais como o YouTube, Instagram, Twitter, Facebook e Tiktok, que possuem ferramentas que permitem ao usuário fazer transmissões de vídeo por meio de sua conta pessoal, possibilitando que os seguidores dessa pessoa/canal/instituição possam acessar e acompanhar em tempo real o conteúdo que está sendo transmitido. Uma característica marcante desse tipo de transmissão é seu poder de interatividade, uma vez que aqueles que a acompanham podem fazer comentários simultaneamente por mensagens registradas na aba de conversa (chat) e registrar reações de contentamento/descontentamento (por meio de um botão de curtidas/descurtidas), além de também poder acompanhar e interagir com as atividades/registros/opiniões dos demais espectadores, conferindo assim dialogicidade a esse processo.

No campo da educação essa estratégia tem sido muito utilizada por educadores (as) e pesquisadores (as) para tratar de questões diversas, evidenciando a riqueza que mencionamos acima. 
Assim esta estratégia tem se mostrado viável, possibilitado a continuidade dos diálogos e ponderações coletivas, entretanto, temos o receio de que a riqueza dessas trocas e análises se perca em meio ao tempo e a diversidade de publicações espalhadas nas redes sociais, incorrendo no risco de que se dissolvam na lógica da velocidade digital.

As discussões acerca dessa temática têm remetido a questões emergentes e de suma importância considerando a drasticidade das mudanças, a imprevisibilidade de 'normalização' dessa situação e as consequências que essas proposições, bem como os impactos delas decorrentes, podem gerar para a Educação nacional.

Nesse sentido, justificam-se registros como este que ora apresentamos, que tem sobretudo, o objetivo de localizar tais iniciativas no tempo e no espaço virtual, sistematizando-as ao registro escrito como meio de buscar garantir que assim resistam ao tempo, conferindo, portanto, materialidade à lives com temáticas de educação realizadas nesse período e possibilitando que essas tantas análises feitas em tempo real, em conjunto com outros registros, auxiliem na composição de um acervo documental capaz de dar visibilidade a hiatos existentes entre o que vem sendo proposto e divulgado no âmbito das políticas públicas de educação em tempos de pandemia e o que vem sendo percebido e observado sob a ótica dos atores envolvidos nesse processo. Ressaltamos que tais hiatos ampliaram-se substancialmente, sendo evidenciados especialmente a partir do período vivenciado na prática a partir de 17 de março de 2020, quando foi decretada a suspensão das atividades escolares presenciais e autorizadas ações de ensino remoto².

Ao pensarmos em ensino remoto, muitas são as preocupações, especialmente ao associarmos esse termo com a ideia de EAD (ensino a distância), tema que mesmo antes da pandemia já habitava as pautas políticas de organização da educação básica do atual governo, gerando desde sempre muitos debates e controvérsias 3 .

\footnotetext{
${ }^{2}$ Cabe esclarecer que nossa busca teve o recorte inicial de tempo planejado em fevereiro de 2020, sob a hipótese de que as reflexões tivessem iniciado naquele período, mas contrariando nossa hipótese, o retorno da busca só gerou resultado a partir do final de março. Ao compor a primeira categoria (i) não encontramos nenhuma incidência de lives sobre o tema da educação infantil nos meses de fevereiro de março, do mesmo modo que na segunda categoria (ii) as duas primeiras lives localizadas foram transmitida em 30 de março de 2020, o que nos levou a inferir que, diferentemente do que supusemos inicialmente, as discussões e reflexões sobre esse assunto só tiveram início após o decreto que determinou a suspensão de aulas e atendimento presenciais nas escolas, o que incorreu na alteração da data inicial desta pesquisa para 17 de março de 2020, muito embora para nossa tristeza, essa ausência também nos deixe indícios quanto a uma possível falta de planejamento ou início tardio das reflexões, uma vez que essa é uma problemática que já vinha sendo discutida em esfera mundial desde meados de dezembro de 2019, quando os casos de covid-19 começaram a se multiplicar na china e no mundo todo.
}

${ }^{3}$ Vide nesse sentido os inúmeros debates feitos acerca da reforma do ensino médio e na proposição de que parte da carga horária pudesse ser oferecida a distância em modalidade EAD. Veja Lei I3.4I5/I7. 
Tais preocupações aumentam ainda mais e multiplicam-se ao pensarmos nas possibilidades de ensino remoto para a educação infantil, situação inédita até então, que carece, a nosso ver, de um olhar detido para as especificidades da infância, o que implica em muitas ponderações para além do que qualquer definição governamental simplista possa dar conta, incluindo no bojo desses encaminhamentos os atores protagonistas e principais interessados na assertividade desses processos, quais sejam: educadores, famílias e crianças, aqui compreendidos como sujeitos de direito a quem deve ser conferido poder de narração e participação como princípio de aprimoramento nas ações desse período da história que vivemos.

Desse modo, tendo como aporte os estudos da infância que compreendem a pedagogia, a antropologia e a sociologia da infância, consideramos a premência de focarmos nosso olhar nas crianças e suas infâncias, inclusive nas propostas voltadas para a educação infantil planejadas e desenvolvidas nesse cenário sem precedentes de educação não presencial no contexto da pandemia, e por essa razão elegemos focalizar lives que tratem de 'educação infantil no contexto da pandemia' para a pesquisa de busca e sistematização dos resultados, com vista a encurtar as distâncias entre tempo e espaço ${ }^{4}$. Considerando a própria dinâmica da internet, na qual os agrupamentos sociais estão dispostos em rede, aqui do mesmo modo, as transmissões de vídeo localizadas foram categorizadas conforme a perspectiva declarada pelos seus preletores e por outras características que detalharemos mais adiante.

\section{Lives tratando de educação infantil disponíveis no Youtube: de onde partimos até o registro dos resultados que encontramos}

Para esta pesquisa utilizamos como referência metodológica a netnografia, abordagem preconizada por Kozinets (20।4), que prevê a utilização de comunicações mediadas por computador como fonte de dados para chegar à compreensão e à representação etnográfica de um fenômeno cultural na, pela e para a internet.

A metodologia da netnografia, inicialmente explorada pela área de Marketing (Kozinets, 1998, 2002) e Comunicação (Hine, 2004), vem ganhando espaço em outras áreas do conhecimento, como a Educação, uma vez que essa abordagem possibilita estudos de fóruns de discussão, grupos de notícias, blogs, redes sociais, comunidades online, entre outros espaços online, o que se torna muito profícuo para os tempos atuais e para o levantamento que aqui nos propusemos a realizar.

\footnotetext{
${ }^{4}$ Registro aqui a gratidão a Ana Lúcia Goulart de Farias, que tem sido parceira constante em diálogos e reflexões que se dão por contato telefônico madrugada afora. Nessas reflexões percebemos juntas a importância de conferir materialidade as lives realizadas nesse período, e foi dessa percepção que nasceu o desejo de publicitar essa contribuição, que ora apresento.
}

Olhar de professor, Ponta Grossa, v. 24, p. I-I8, e-16055.075, 202 I.

Disponível em <https://revistas2.uepg.br/index.php/olhardeprofessor> 
Segundo Kozinets, o fenômeno estudado pela netnografia pode ser caracterizado em pelo menos 3 (três) categorias: (i) do presencial ao virtual: refere-se ao termo na internet - pode ter início no mundo presencial e alcançar o mundo virtual, (ii) do virtual ao presencial: refere-se ao termo pela internet- pode começar no mundo virtual e se propagar para o mundo presencial e (iii) exclusivamente virtual: refere-se ao termo para a internet- se estabelece exclusivamente no universo virtual.

Nossa proposta se encaixa, portanto, na segunda categoria preconizada pelo estudioso, uma vez que buscamos por interações feitas por meio de transmissões de vídeo ao vivo na internet, com o intuito de que essas discussões/reflexões/ ponderações alcancem a realidade fora das telas e se propague por meio de pesquisas feitas no universo acadêmico real.

Nesse sentido, considerando a maior abrangência e o potencial de veiculação de registros em vídeos ao vivo, utilizamos o youtube como fonte de busca, a partir do termo chave: 'Educação infantil em tempos de pandemia'. Importante ressaltar que configuramos a busca para que apresentasse registros contendo a totalidade e a parcialidade das palavras utilizadas, o que resultou em uma abrangência maior de lives registradas na temática.

Segundo os preceitos da netnografia, há decisões fundamentais a serem tomadas antes do primeiro contato com uma comunidade online. Assim o primeiro procedimento refere-se à entrée cultural, na qual são tomadas decisões sobre a preparação para o trabalho de campo, como a identificação da comunidade online, temas e questões que serão investigadas, dentre outras questões; o pesquisador deve deixar claro o ponto de observação ao fazer as considerações sobre seu objeto, pois seu posicionamento pode interferir na forma como os dados serão coletados e analisados.

Neste sentido optamos por utilizar como recorte de tempo a data inicial o dia 17 de março de 2020, quando foi decretada a suspensão das aulas presenciais e autorização da substituição por aulas em meios digitais e, portanto, discutidas as medidas educacionais do período de isolamento social que se antevia. Como data final fixamos o dia 12 de jun. de 2020, o que não significa que as publicações tenham se esgotado, muito pelo contrário, a incidência de lives sobre o tema tem ficado cada vez mais efusiva; Assim a data limite que fixamos se remete diretamente ao prazo para encerramento desse registro e encaminhamento para publicação estabelecido por essa revista, que tornou-se o meio escolhido, por abrir esse espaço tão importante de contribuição mais aberta e fluída, confluindo assim com nossos anseios e objetivos.

Ao todo a busca realizada, localizou 78 lives que tratam do tema, as quais foram organizadas em duas categorias, conforme suas características, delimitadas a partir do conteúdo individual analisado em cada transmissão, tendo como critério principal a observação quanto ao que se defendia. Desse modo a primeira categoria (i) foi composta pelas lives que traziam indicativos de ter como premissa a defesa/ divulgação (direta ou indireta) de ideias e concepções privadas, e a segunda categoria (ii) foi 
composta pelas lives que tiveram veiculação única com interesse de análise da conjectura atual, discutindo a educação infantil no contexto da pandemia sob um olhar crítico, apartidário e tendo como objetivo maior a defesa das crianças e suas infâncias nesse contexto. Na sequência apresentamos os achados de cada categoria.

\section{Resultados que encontramos: contribuições para a composição do cenário atual da educação infantil que se apresenta}

Definidas na primeira categoria (i) listamos as lives que estavam submetidas e/ou sendo patrocinadas por algum órgão com interesses específicos. Assim nesta categoria listamos transmissões de vídeo veiculadas por empresas e instituições privadas (fossem de educação ou formação docente), bem como aquelas que estavam sendo patrocinadas por editoras e correlatos. Também encaixamos nessa categoria aquelas em cujos preletores trazer em seu histórico participações e/ou defesas a procedimentos e propostas, como a BNCC para a educação infantil ou ainda propostas feitas por esferas governamentais desta ou de outra gestão. E incluímos ainda, pela mesma razão, lives que trouxessem entre os convidados pesquisadores que estão atuando como assessores municipais ou estaduais, e também as que tenham sido promovidas por candidatos ou membros políticos.

Categorizadas nesse eixo localizamos 42 lives publicadas, cujos endereços virtuais estão listados como apêndice $A$ ao final desse registro, organizados por ordem cronológica, atendendo a finalidade de composição de acervo documental para pesquisas futuras. Cabe esclarecer que embora nossa busca tenha o recorte de tempo com início em março, ao compor essa categoria somente encontramos transmissões de vídeo com o tema da educação infantil a partir do mês de abril, sendo a primeira transmissão localizada em 08 de abril de 2020. Dentre tantos ensaios e problematizações, destacamos especialmente as que apresentam ponderações no tocante as concepções e as alternativas que têm sido vislumbradas para a continuidade de ações e processos educativos nesse período de distanciamento social, incorrendo assim nas mais diversas propostas de ensino remoto que vem sendo implantadas por Redes municipais, estaduais e privadas de todo o país.

Definidas na segunda categoria (ii) listamos, conforme já anunciamos, lives que tiveram veiculação única com interesse de análise da conjectura atual, discutindo a educação infantil no contexto da pandemia sob um olhar crítico, apartidário e tendo como premissa principal a defesa das crianças e suas infâncias nesse contexto, compreendendo que as políticas e proposições (quais sejam) devem considerar e fazer valer na prática o respeito às crianças enquanto sujeitos históricos de direito - sendo o maior deles o direito a infância.

Desse modo essa categoria compreendeu lives promovidas por instâncias e organizações compostas por membros da sociedade civil em defesa da criança, como é o caso dos fóruns de 
educação infantil. Foram também categorizadas nesse eixo lives realizadas por universidades e por pesquisadores da área da educação que se reuniram por iniciativa própria. Há ainda nessa categoria lives que reuniram educadores e gestores para apresentar relatos de experiência e também pessoas que tem olhado para as questões da infância nesse período de pandemia por outras perspectivas, de modo a congregar esforços para que múltiplas questões relacionadas ao desenvolvimento biopsicossocial das crianças sejam consideradas e respeitadas.

Categorizadas nesse eixo localizamos 36 lives publicadas, cujos endereços virtuais também estão listados ao final desse registro (apêndice B), com vistas a compor de forma conjunta com a categoria anterior, o acervo documental que ensejamos sistematizar.

\section{Considerações finais/conclusões}

Ao analisarmos brevemente o conteúdo dos vídeos para poder categorizá-los, ficou perceptível na primeira categoria (i), embora não possamos fazer uma afirmação contundente (o que careceria de uma pesquisa específica para esse fim), que as concepções defendidas ainda que contando por vezes com pesquisadores da área, possam estar de certo modo comprometidas com os objetivos das agências promotoras e/ou das instituições em que atuam esses interlocutores e, portanto, passíveis de estarem sendo submetidas a interesses particulares, o que pode torná-las tendenciosas e até em certos casos incongruentes com o que a realidade revela do ponto de vista da criança e das infâncias. Por essa razão não negamos sua importância ou potencial de contribuição mas deixamos aqui registrada a ressalva para que quem deseje fazer uso desses conteúdos em suas pesquisas considere essa ponderação ao proceder a análise.

Já na análise dos conteúdos que caracterizaram as lives que foram enquadradas na segunda categoria (ii), tivemos a grata surpresa de observar que muitas pessoas, de diferentes áreas do conhecimento, têm se reunido para olhar conjuntamente para as crianças e suas infâncias nesse período de pandemia. Ao observar os participantes das lives que listamos encontramos, além de estudantes e pesquisadores da área da educação, jornalistas, psicólogos, representantes da área jurídica e até mesmo mães que vem deixando registrado aspectos, percepções e preocupações extremamente relevantes nesse tema. Destacamos ainda uma das lives que além de educadores e pesquisadores, conta também com a participação inédita das crianças, que somam sua mais que necessária voz a discussão.

Esperamos assim que tais agrupamentos possam funcionar tanto no espaço online, como coexistir fora dele, no espaço off-line, garantindo que o caráter investigativo e de observação da realidade possibilitados pela riqueza dessas trocas e análises sejam preservados tanto no presente quanto em um futuro próximo, de modo que nos permitam compor um quadro analítico o mais 
verossímil possível da realidade atual das crianças e das infâncias no cenário da educação infantil que se apresenta nesse período em que perdure a quarentena, do mesmo modo que permita observar os impactos que podem gerar/estar gerando para a Educação infantil no contexto nacional.

Aspiramos ainda que este quadro possa ser usado como recurso para entender como a injustiça e a desigualdade social sistêmica ocorrem em uma base multidimensional, tornando-se assim ferramenta de compreensão e proposição de mudança de tudo que será preciso rever posteriormente (ou já está sendo evidenciado) do ponto de vista da interseccionalidade.

\section{Referências}

HINE, C. Etnografía virtual. México: Editorial UOC, 2004. Disponível em: https://seminariosocioantropologia.files.wordpress.com/20I4/03/hine-christine-etnografia-virtualuoc.pdf. Acesso em 12 de junho de 2020.

KOZINETS, R. V. Netnografia: Realizando pesquisa etnográfica online. Porto Alegre: Penso, 2014.

\section{APÈNDICE A - LIVES CATEGORIA (i)}

Título: Escutar as crianças em tempos de quarentena

Participantes: Josiane Pareja, Rita Paula Cardoso e Lica (Eliana Sisca)

Canal/Entidade promotora: Atêlie Carambola Escola de Educação Infantil

Transmitido ao vivo em: 08 de abr. de 2020

Link de acesso: https://youtu.be/49qTH6GnIPo

Título: Como atender as crianças da educação infantil nesse momento da pandemia

Participantes: Barbiere (SINEPE NOPR), Regina Shudo e Jane Haddad

Entidade promotora: $O$ vídeo foi transmitido pelo canal 'SINEPE NOPR' que é vinculado ao sindicato dos estabelecimentos particulares

Transmitido ao vivo em: 09 de abr. de 2020

Link de acesso: https://youtu.be/ZROYynePhWY

Título: Quarentena: Como fica a educação infantil

Participantes: Andrea Miranda

Canal/Entidade promotora: Este vídeo foi transmitido pelo canal PPD, que está vinculado à empresa de nome homônimo, que atua na área de assessoria educacional, venda de cursos e etc Transmitido ao vivo em: 09 de abr. de 2020

Link de acesso: https://youtu.be/j-r_RGGwjUc

Título: Resumo da live Estratégias para a El em tempos de distanciamento social

Participantes: Paulo Sergio Fochi

Canal/Entidade promotora: Canal pessoal Paulo Sergio Fochi

Transmitido ao vivo em: 13 de abr. de 2020

Link de acesso: https://youtu.be/9iwf2xuwyp0

Título: Live mágica/ Educação Infantil em tempos de coronavírus

Olhar de professor, Ponta Grossa, v. 24, p. I-I8, e-I6055.075, 202 I.

Disponível em <https://revistas2.uepg.br/index.php/olhardeprofessor> 
Participantes: Gabi Alkmim, Katheen Arcanjo

Canal/Entidade promotora: Canal estante magica, vinculado a empresa de nome homônimo que atua na organização e venda de projetos de leitura.

Transmitido ao vivo em: 14 de abr. de 2020

Link de acesso: https://youtu.be/Q4miM9tkHGY

Título: Como estão as crianças da Educação Infantil em tempos de pandemia com Jane Haddad e Regina Shudo

Participantes: Paulo (Ferraz eventos), Regina Shudo, Jane Haddad,Priscila Machado e Bartolomeu Moura

Canal/Entidade promotora: Canal Amaná Educacional- Regina Shudo

Transmitido ao vivo em: 17 de abr. de 2020

Link de acesso: https://youtu.be/TboUdSPQxjs

Título: $O$ cenário da educação infantil em tempos de pandemia

Participantes: Paulo (Ferraz eventos), Regina Shudo, Alessio Costa Lima, Jane Patricia Haddad

Entidade promotora: Ferraz eventos

Transmitido ao vivo em: 20 de abr. de 2020

Link de acesso: https://youtu.be/NI q6d88YvGE

Título: Como pensar a Educação infantil em tempos de isolamento social

Participantes: Luciana (editora Opet)

Canal/Entidade promotora: Editora Opet

Transmitido ao vivo em: 23 de abr. de 2020

Link de acesso: https://youtu.be/hls8rZISXkg

Título: Educação das Infâncias em tempos de isolamento social

Participantes: Janaína Carrico e Andrea Carla

Canal/Entidade promotora: canal Undime- RN

Transmitido ao vivo em: 23 de abr. de 2020

Link de acesso: https://youtu.be/tBs2T28CEa8

Título: Live c/ Carolina Freitas- Desafios dos pais da educação infantil no contexto da pandemia.

Participantes: Neide Santos e Carolina Freitas

Canal/Entidade promotora: Espaço Crescer em família

Transmitido ao vivo em: 24 de abr. de 2020

Link de acesso: https://youtu.be/WJASXAyZNe8

Título: LIVE - Como cuidar das crianças em tempos de pandemia?

Participantes: Luccas Couto (Sunny Brinquedos), Paula Bertapeli e Aline Matias

Canal/Entidade promotora: Canal Descubra o mundo Brincando- Sunny Brinquedos

Transmitido ao vivo em: 28 de abr. de 2020

Link de acesso: https://youtu.be/gFqts I Ru3QM

Título: Educação infantil em tempos de pandemia

Participantes: Márden de Paula, Heloisa Baldo e Fernanda Clímaco

Entidade promotora: $O$ vídeo foi transmitido diretamente do canal do professor Márden, entretanto na descrição os convidados, incluindo o próprio Márden são apresentados como assessores pedagógicos do Bernoulli sistema de ensino

Transmitido ao vivo em: 05 de mai. de 2020

Link de acesso: https://youtu.be/ASFyN0cF-V4

Olhar de professor, Ponta Grossa, v. 24, p. I-I8, e-16055.075, 202 I.

Disponível em <https://revistas2.uepg.br/index.php/olhardeprofessor> 
Título: Educação infantil em tempos de distanciamento

Participantes: Dani Sá (DL cursos), Isabel Ceron e Elen Padilha

Canal/Entidade promotora: Canal DS CURSOS DANI SÁ

Transmitido ao vivo em: 06 de mai. de 2020

Link de acesso: https://youtu.be/f5kI_AgDsZ0

Título: Educação Infantil. Como trabalhar com os pequenos em época de pandemia

Participantes: Paulo (Ferraz eventos), Jane Haddad, Regina Shudo e Lucia Aurélia

Canal/Entidade promotora: Ferraz eventos e treinamentos

Transmitido ao vivo em: 08 de mai. de 2020

Link de acesso: https://youtu.be/BnzO9UEyy3g

Título: LIVE |É possível uma Educação Infantil a distância?

Participantes: Beatriz Cardoso, Rogério Morais, André Lázaro e Miguel Thompsonn

Canal/Entidade promotora: Editora Moderna

Transmitido ao vivo em: 08 de mai. de 2020

Link de acesso: https://youtu.be/ZAQDCpmneyE

Título: Diálogo Marista - Educação Infantil: desafios emergentes no contexto do isolamento social Participantes: Lourdes Atié, Maria Carmen Barbosa, Rita Coelho, Zilma de Oliveira e Elaine Castello

Canal/Entidade promotora: UMBRASIL- União Marista do Brasil

Transmitido ao vivo em: 12 de mai. de 2020

Link de acesso: https://youtu.be/P4OHqWEROzw

Título: Depois da Pandemia: A reinvenção da educação infantil | Órama Investimentos

Participantes: Hugo Daniel Azevedo e Ana Paula Pontes

Canal/Entidade promotora: ORAMA investimentos

Transmitido ao vivo em: 13 de mai. de 2020

Link de acesso: https://youtu.be/5pnQAs6GW4k

Título: Ensino remoto na Educação Infantil

Participantes: Eduardo Deschamps

Canal/Entidade promotora: Canal TV SINEPE- RS, vinculado ao sindicato do ensino privado

Transmitido ao vivo em: 14 de mai. de 2020

Link de acesso: https://youtu.be/X4H8Bsdajpg

Título: Educação infantil em tempos de pandemia

Participantes: Tarcísio Motta, Ana Carolina Martins, Barbara Amado e Joselma Brito

Entidade promotora: $O$ vídeo foi transmitido diretamente do canal de Tarcísio Motta, que atualmente é vereador da cidade do Rio de Janeiro

Transmitido ao vivo em: 15 de mai. de 2020

Link de acesso: https://youtu.be/CCgqQ5QfYHI

Título: Educação Infantil em tempos de pandemia

Participantes: Diumberto Freitas (Sec. Educação), Conceição Melo, Cristianny Portela, Geane Rebouças

Canal/Entidade promotora: Canal Seduc ICAPUI, vinculado a Prefeitura de Icapuí

Transmitido ao vivo em: 15 de mai. de 2020

Link de acesso: https://youtu.be/E6bBVXzcEZQ

Olhar de professor, Ponta Grossa, v. 24, p. I-I8, e-16055.075, 202 I.

Disponível em <https://revistas2.uepg.br/index.php/olhardeprofessor> 
Título: Live Solidária - Crianças em tempo de pandemia

Participantes: Priscila Pincos e Carla Marçal

Canal/Entidade promotora: Sesc RJ, vinculado ao projeto mesa Brasil

Transmitido ao vivo em: 15 de mai. de 2020

Link de acesso: https://youtu.be/L7hCyUGy9pw

Título: \#CompartilheBoasPráticas [0I] - A Pandemia e o desafio da Educação Infantil

Participantes: Alana (SINEPE), Rosana Rego Cairuga

Canal/Entidade promotora: TV SINEPE - RS, vinculado ao sindicato do ensino privado

Transmitido ao vivo em: 18 de mai. de 2020

Link de acesso: https://youtu.be/EvB2sBxzvSc

Título: Perspectivas para o retorno na Educação Infantil - Rita Coelho

Participantes: Paulo Fochi e Rita Coelho

Canal/Entidade promotora: Canal pessoal de Paulo Sergio Fochi

Transmitido ao vivo em: 18 de mai. de 2020

Link de acesso: https://youtu.be/vSxdCajUG5Y

Título: Educação infantil e suspensão de atendimento. Como ficam as crianças pequenas na pandemia?

Participantes: Gunga

Canal/Entidade promotora: Canal Respeitar é Preciso- Instituto Vladimir Herzog

Transmitido ao vivo em: 18 de mai de 2020

Link de acesso: https://youtu.be/9I-DWYbwf5A

Título: Live - Desafios da Educação Infantil e do $I^{\circ}$ ano EFI em tempos de coronavírus

Participantes: Fabi Mamone, Michelle Taborna e Ane Vigiano

Canal/Entidade promotora: Conquista Solução Educacional

Transmitido ao vivo em: 21 de mai. de 2020

Link de acesso: https://youtu.be/9VBJNV3oVZA

Título: Live - Educação Infantil: conversas para quem gosta de ensinar

Participantes: Michele Taborda e Ana Paula Santana

Canal/Entidade promotora: Conquista Solução Educacional

Transmitido ao vivo em: 27 de abr. de 2020

Link de acesso: https://youtu.be/Urip2_0r6O0

Título: Roda de conversa: o que a Educação Infantil está fazendo em tempos de pandemia

Participantes: Cidia Silveira, Daniela Ilha, Uidia Rocha, Maia, Ângela e Carol

Canal/Entidade promotora: Canal Formação de professores SME- Prefeitura de Canoas

Transmitido ao vivo em: 28 de mai. de 2020

Link de acesso: https://youtu.be/E3-__GEUX-8

Título: Desafios da educação infantil em tempos de pandemia

Participantes: Juliana Camarão e Duarte Junior

Canal/Entidade promotora: $O$ vídeo foi transmitido diretamente do canal de Duarte Júnior, que atualmente é Deputado Estadual

Transmitido ao vivo em: 28 de mai. de 2020

Link de acesso: https://youtu.be/dxaJ7E5Rsb8

Olhar de professor, Ponta Grossa, v. 24, p. I-I8, e-16055.075, 202 I.

Disponível em <https://revistas2.uepg.br/index.php/olhardeprofessor> 
Título: Bate Papo: Arte e educação infantil a partir da pandemia: possíveis transformações

Participantes: Ana Paula Yazbek (Espaço educação) e Maria Paula Zurawski

Canal/Entidade promotora: Canal Espaço Educação Infantil

Transmitido ao vivo em: 0 I de junho de 2020

Link de acesso: https://youtu.be/Q7iJNP46AMU

Título: Educação infantil em tempos de pandemia

Participantes: Cyntia (consultora SAS), Daniela Cristina Siqueira, Flavia Vano e Fernanda (colégio satélite)

Canal/Entidade promotora: Colégio Satélite

Transmitido ao vivo em: 02 de jun. de 2020

Link de acesso: https://youtu.be/XqtUU5uOqDc

Título: Webinar EP 45: Os desafios da educação infantil durante e pós-pandemia (com Maria Thereza Marcílio)

Participantes: Carlos Sanches e Maria Thereza Marcilio

Canal/Entidade promotora: Canal PVE- Instituto Votorantin

Transmitido ao vivo em: 03 de jun. de 2020

Link de acesso: https://youtu.be/7sN4rvZVW2I

Título: Os desafios da Educação Infantil em tempos de Pandemia

Participantes: Amanda Hottes

Canal/Entidade promotora: CEAS CRECHE ESCOLA

Transmitido ao vivo em: 05 de jun. de 2020

Link de acesso: https://youtu.be/nKMA3MA83_E

Título: Case de sucesso na educação infantil em tempos de pandemia - SMEC Tramandaí

Participantes: Professora não identificada

Canal/Entidade promotora: Canal pessoal de Andrios Bemfica, vinculado a SMEC Tramandaí

Transmitido ao vivo em: 5 de jun. de 2020

Link de acesso: https://youtu.be/QKhi5S2v668

Título: Educação infantil em tempos de pandemia

Participantes: Débora Brondani (TCE), Rita Coelho, Paulo Fochi

Entidade promotora: Tribunal de conta do Estado RS

Transmitido ao vivo em: 05 de jun. de 2020

Link de acesso: https://youtu.be/VGrZTxMqUlg

Título: Profissionais de Educação Infantil em Tempos de Pandemia

Participantes: Samuel Nascimento, Elaine Cristina, Cleonice Santos, Thais Cherubini

Canal/Entidade promotora: Canal TecPed- Equipe Tecnopedagógica, vinculado ao departamento de apoio pedagógico da Secretaria de Educação de Barueri

Transmitido ao vivo em: 09 de jun. de 2020

Link de acesso: https://youtu.be/MJwECJZSNs8

Título: 10/06/2020 - Manhã/Tarde - Aprendizagens Inovadoras Educação Infantil

Participantes: Juliane Menezes, Karina Perez e Sheila Arantes

Canal/Entidade promotora: Canal LEDI- Laboratório de Educação Digital Interativa, vinculado a secretaria de educação e cidadania de São José dos Campos

Transmitido ao vivo em: 09 de jun. de 2020

Link de acesso: https://youtu.be/dB6TiBPrXEk 
Título: Websérie - Refletindo as Políticas Públicas de Educação Infantil durante a pandemia

Participantes: Maria Thereza Marcilio, Rita Coelho

Canal/Entidade promotora: Canal Avante ONG

Transmitido ao vivo em: 10 de jun. de 2020

Link de acesso: https://youtu.be/Etbxk7iji7E

Título: Cuidar e educar na educação infantil em tempos de pandemia

Participantes: Lauri Cericato (Diretor editorial Saber) e Zilma Ramos de Oliveira

Entidade promotora: $O$ vídeo foi transmitido pelo canal e-docente, que é vinculado a editora

Saber, apresentada por Lauri como responsável pelas publicações didáticas e de letratura dos selos

Ática, Saraiva e Scipione.

Transmitido ao vivo em: 10 de jun. de 2020

Link de acesso: https://youtu.be/XZRmYu2wZmU

Título: A educação infantil em tempos de pandemia

Participantes: Cris Cristo

Canal/Entidade promotora: Canal pessoal \#MÂEDETRÊS Cris

Transmitido ao vivo em: 10 de jun. de 2020

Link de acesso: https://youtu.be/592Bpq_JwJY

Título: LIVE - CONVERSANDO SOBRE EDUCAÇÃO INFANTIL \#2

Participantes: Elizabeth Conte, Fabiana Meirelles,Rosa Cavalcante, Cláudia Chebabi

eAdriana Ramos (C+).

Canal/Entidade promotora: Canal convivere mais- Assessoria e formação

Transmitido ao vivo em: II de jun. de 2020

Link de acesso: https://youtu.be/Xlmst-8nkjU

Título: A criança e a escola de educação infantil

Participantes: Letícia Fernandes e Adriana di Mambro

Canal/Entidade promotora: Centro Lúdico de Interação e Cultura CLIC

Transmitido ao vivo em: I I de jun. de 2020

Link de acesso: https://youtu.be/ IIWS6 I qidX4

Título: A educação infantil e a pandemia

Participantes: Filó, Eni Pereira de Souza, Marcos R. Coutinho, Luci Guidio

Canal/Entidade promotora: Setorial Municipal de Educação PT São Paulo

Transmitido ao vivo em: 12 de jun. de 2020

Link de acesso: https://youtu.be/vGuvOHk7izo

\section{APÊNDICE B - LIVES CATEGORIA (ii)}

Título: Como a pandemia de coronavírus afeta a educação de crianças no Brasil

Participantes: Denise Carreira, Mariana Luz e Vagner de Alencar

Canal/Entidade promotora: JEDUCA- Associação de jornalistas de educação

Transmitido ao vivo em: 30 de mar. De 2020

Link de acesso: https://youtu.be/NIGb_HcXcqo

Título: Regime especial de atividades não presenciais

Olhar de professor, Ponta Grossa, v. 24, p. I-18, e-16055.075, 202 I.

Disponível em <https://revistas2.uepg.br/index.php/olhardeprofessor> 
Participantes: Maíra Dourado

Canal/Entidade promotora: Escutatória- Para pensar a educação infantil

Transmitido ao vivo em: 30 de març. De 2020

Link de acesso: https://youtu.be/SK8ixkgKUKw

Título: A PANDEMIA E O EAD NA EDUCAÇÃO INFANTIL DA REDE MUNICIPAL DE SÃO PAULO

Participantes: Emilio Rodriguez, Michelle Faria e Natalia Cardia

Canal/Entidade promotora: Canal Jornalistas Livres

Transmitido ao vivo em: 19 de abr. de 2020

Link de acesso: https://youtu.be/8OhmWX9cMuU

Título: Relatos de Experiências da Educação Infantil em tempos de pandemia

Participantes: Jeiviane e Greice Dorta

Canal/Entidade promotora: Blog BaguncEI

Transmitido ao vivo em: 22 de abr. de 2020

Link de acesso: https://youtu.be/zsLMIds-XZM

Título: CORONAVÍRUS: OS DIREITOS DA CRIANÇA E DO ADOLESCENTE EM TEMPOS DE PANDEMIA

Participantes: Carla Soraya e Tati Andrade

Canal/Entidade promotora: TV Ceará

Transmitido ao vivo em: 22 de abr.de 2020

Link de acesso: https://youtu.be/zkv5zIIRpM

Título: Podcast 07 | Crianças e seus direitos: Educação Infantil Presente - Célia Serrão

Participantes: Marcia Gobbi e Célia Serrão

Canal/Entidade promotora: Canal em movimentos

Transmitido ao vivo em: 23 de abr. de 2020

Link de acesso: https://youtu.be/FfJElzjosdM

Título: A educação infantil e suas possibilidades durante a pandemia

Participantes: Luiz Miguel Garcia, Maria Regina de Passos e Beatriz Abuchaim

Canal/Entidade promotora: Conviva Educação

Transmitido ao vivo em: 24 de abr. de 2020

Link de acesso: https://youtu.be/OrQfzYc78aw

Título: Educação Infantil - I Ciclo de encontros: Educação e formação em tempos e cenários de pandemia

Participantes: Itale Cericato, João do Prado, Celia Serrão, Renata Cristina Dias Oliveira, Jessica Blasques da Silva, Edivaldo dos Reis Oliveira Filho

Canal/Entidade promotora: PRODOC UNIFESP

Transmitido ao vivo em: 0 I de mai. de 2020

Link de acesso: https://youtu.be/QR4PJeRcB9|

Título: A Educação Infantil no contexto da pandemia. Com a prof. Ana Christina de Abreu Araújo Participantes: Ana Christina de Abreu Araújo

Canal/Entidade promotora: canal tvunicarioca

Transmitido ao vivo em: 05 de mai. de 2020

Link de acesso: https://youtu.be/0bbG89jD4EE

Olhar de professor, Ponta Grossa, v. 24, p. I-18, e-16055.075, 2021.

Disponível em <https://revistas2.uepg.br/index.php/olhardeprofessor> 
Título: Educação infantil durante a pandemia é tema de entrevista

Participantes: Giulia Pontes e Karina Fasson

Canal/Entidade promotora: JornalismoESPMSP

Transmitido ao vivo em: 07 de maio de 2020

Link de acesso: https://youtu.be/knxD6vHyets

Título: Live: Proteção Integral de Crianças e Adolescentes em tempos de pandemia

Participantes: Marina de Porponi Vaz, lolete Ribeiro da Silva e Deise Cesar Franco Bernardes

Canal/Entidade promotora: Conselho Federal de Psicologia

Transmitido ao vivo em: 07 de mai. de 2020

Link de acesso: https://youtu.be/pyZGexEGMxU

Título: Webnário Internacional - A Creche pós Pandemia - Planejamento e Acolhimento

Participantes: Leila Oliveira, Ana Carol Thomé, Daniela Silva, Mari Polke, Gabriela Yrala

Canal/Entidade promotora: Canal Insaberes- Com Leila Oliveira

Transmitido ao vivo em: 10 de mai. de 2020

Link de acesso: https://youtu.be/ECS6Vxzmllg

\section{Título: O PAPEL DAS INSTITUIÇÕES DE EDUCAÇÃO INFANTIL EM TEMPOS DE} DISTANCIAMENTO SOCIAL

Participantes: Samantha Lima, Sabrina Garcez, Ariete Brusio, Laiser Melo e outros membros participantes do fórum gaucho

Canal/Entidade promotora: Canal Fórum Gaúcho de Educação Infantil

Transmitido ao vivo em: II de mai. de 2020

Link de acesso: https://youtu.be/VfVYaabSVIU

Título: Reunião Aberta (Virtual) FEIPAR - Educação Infantil no Parecer do CNE sobre a Pandemia Participantes: Angela Coutinho, Carla Vilar, Soeli Pereira, Juliana Moralles Louvison, Juliana, Cassiana Magalhães, Renata Oliveira Silva

Canal/Entidade promotora: FEIPAR- Fórum de educação infantil do Paraná

Transmitido ao vivo em: 12 de mai. de 2020

Link de acesso: https://youtu.be/l8wQZ-LZOyc

Título: Educação infantil em contexto de pandemia: limites e possibilidades

Participantes: Profa Giseli (FAPIDE), Viviane Reis, Isabel Oliveira

Canal/Entidade promotora: Vídeo transmitido pelo Canal pessoal de Ivandro Menezes, vinculado ao V ciclo de conferencias da FAPIDE (Faculdade Pio Décimo de Canindé do São Francisco),

intitulado a pedagogia no contexto da pandemia;

Transmitido ao vivo em: 20 de mai. de 2020

Link de acesso: https://youtu.be/tlFVU4yThFM

Título: Educação infantil em tempos de pandemia: práticas e reflexões

Participantes: Adriane Mobbs, Eliane Mello e Sueli Figueiredo Wagner

Canal/Entidade promotora: Canal pessoal Eliane Mello

Transmitido ao vivo em: 20 de mai. de 2020

Link de acesso: https://youtu.be/PH7nzmmKoFo

Título: As crianças, a Educação Infantil e a Pandemia

Participantes: Edna Lopes, Fabiana Canavieira e Fábio Hoffmann Pereira

Canal/Entidade promotora: Sinteal Alagoas

Transmitido ao vivo em: 21 de mai. de 2020

Olhar de professor, Ponta Grossa, v. 24, p. I-I8, e-16055.075, 202 I.

Disponível em <https://revistas2.uepg.br/index.php/olhardeprofessor> 
Link de acesso: https://youtu.be/iouYOA6mINO

Título: FAPEI- Fórum do Areste Paraibano de Educação Infantil

Participantes: Normadete de Macedo, Aguifaneide, Katia Campos, Fernanda Leal e Josefa Roseane Nobre, Gina Oliveira

Canal/Entidade promotora: Canal Philippe Araujo

Transmitido ao vivo em: 21 de mai. de 2020

Link de acesso: https://youtu.be/Twcm7K8mVG8

Título: A Educação infantil em tempos de pandemia

Participantes: Adelaide Alves Dias, Célia Maria Vieira dos Santos e Fernanda Mendes Cabral

Canal/Entidade promotora: Forum de educação Infantil da Paraíba

Transmitido ao vivo em: 22 de mai. de 2020

Link de acesso: https://youtu.be/QQyK8JeQrTk

Título: Infância e Educação infantil: desafios da pandemia

Participantes: Daniela Guimarães, Deise Arenhart, Núbia Santos e Patrícia Corsino, Alice

Coutinho e Carolina Sales

Canal/Entidade promotora: Canal pessoal de Patricia Corsino

Transmitido ao vivo em: 24 de mai. de 2020

Link de acesso: https://youtu.be/ZIdSI8YKDKg

Título: Educação de crianças de 0 a 3 anos em tempos de pandemia: desafios e possibilidades

Participantes: Fernanda Almeida

Canal/Entidade promotora: TV UFBA - Congresso virtual UFBA 2020

Transmitido ao vivo em: 25 De mai. de 2020

Link de acesso: https://youtu.be/vt7z76iTu28

Título: Educação infantil em tempos de pandemia

Participantes: Renata Dias, Fernanda Souza e Marcia Gobbi

Canal/Entidade promotora: Fórum Paulista de Educação Infantil

Transmitido ao vivo em: 26 de mai. de 2020

Link de acesso: https://youtu.be/o5Q8OX2goYw

Título: A educação infantil no contexto de (pós) pandemia: narrativas político-pedagógicas em cena Participantes: Marlene Oliveira dos Santos, Nanci Helena Rebouças Franco, Silvanne Ribeiro, Leila da Franca Soares, Daniela Nascimento Varandas, Raquel Alves da Silva Barreto

Canal/Entidade promotora: TV UFBA

Transmitido ao vivo em: 28 de mai. de 2020

Link de acesso: https://youtu.be/aav0zAazOhg

Título: A educação infantil em tempos de pandemia

Participantes: Sumika Freitas, Nathan Moretto, Washington Carneiro e Fabricio Rodrigues

Canal/Entidade promotora: Canal Hora do intervalo

Transmitido ao vivo em: 28 de mai. de 2020

Link de acesso: https://youtu.be/joVdeu7BuME

Título: Expresso 227: Coronavírus, infâncias e desigualdades

Participantes: Marina Pita, Renata Assumpção, Suelaine Carneiro e Edna Araújo

Canal/Entidade promotora: Alana

Olhar de professor, Ponta Grossa, v. 24, p. I-I8, e-I6055.075, 202 I.

Disponível em <https://revistas2.uepg.br/index.php/olhardeprofessor> 
Transmitido ao vivo em: 29 de mai. de 2020

Link de acesso: https://youtu.be/glOBxvaQQvA

Título: Como fica a relação creche - família em tempos de pandemia? - LIVE - 26/05/2020

Participantes: Ana Lucia Goulart de Faria e Fernanda Mendes Cabral

Canal/Entidade promotora: Forum de Educação infantil da Paraiba

Transmitido ao vivo em: 3 I de mai. de 2020

Link de acesso: https://youtu.be/Fmai3367YkA

Título: XONGANI FALA SOBRE A EDUCAÇÃO INFANTIL A DISTÂNCIA EM TEMPOS DE PANDEMIA

Participantes: Xongani

Canal/Entidade promotora: Canal de bens com a vida

Transmitido ao vivo em: 01 de jun. de 2020

Link de acesso: https://youtu.be/wDVxDuXBNZQ

Título: E agora?!? A educação infantil em tempos remotos

Participantes: Geraldo Peçanha de Almeida, Daíne Silva, Felippie (GEPPETE), Barbara Correa,

Eduardo Fofonca

Canal/Entidade promotora: Grupo de pesquisa GEPPETE- UFPR

Transmitido ao vivo em: 03 de jun. de 2020

Link de acesso: https://youtu.be/laLw3hg8Fx0

Título: Educação infantil e as políticas governamentais na pandemia

Participantes: Valdete Côco e Ângela Coutinho

Canal/Entidade promotora: ANPED Nacional

Transmitido ao vivo em: 03 de jun. de 2020

Link de acesso: https://youtu.be/DI7IQZzG49A

Título: CONFERÊNCIA "EDUCAÇÃO INFANTIL E PANDEMIA" - PROFA. DRA. SINARA

ALMEIDA

Participantes: Lino Arlem, Dannielle Costa e Sinara Almeida

Canal/Entidade promotora: ICED UFOPA - canal oficial do Instituto de Ciências da Educação da Ufopa (Universidade Federal do Oeste do Pará)

Transmitido ao vivo em: 04 de jun. de 2020

Link de acesso: https://youtu.be/zEB3fKVHuYo

Título: Contribuições de Profissionais da Educação Infantil em Tempos de Pandemia

Participantes: Ayla Bragança, Maria Consuelo, Joice Rosa, Mariana Marins, Glauciene Ribeiro e

Ludmila Franco

Canal/Entidade promotora: Canal pessoal de Alan Santos

Transmitido ao vivo em: 04 de jun. de 2020

Link de acesso: https://youtu.be/RIWy54R_X0s

Título: LIVE - Cuidados e atividades para crianças durante a pandemia

Participantes: Karla Azevedo, Antonio Ítalo e Miliana Sampaio.

Canal/Entidade promotora: Canal UNITINs- Universidade Estadual do Tocantins

Transmitido ao vivo em: 04 de jun. de 2020

Link de acesso: https://youtu.be/FXSyGYLD2cl

Título: Ensino remoto na Educação Infantil

Olhar de professor, Ponta Grossa, v. 24, p. I-18, e-16055.075, 2021.

Disponível em <https://revistas2.uepg.br/index.php/olhardeprofessor> 
Participantes: Rute Regis de Oliveira da Silva, Maristela Mosca, Márcia Maria Gurgel, e Alexandre Soares Gomes.

Canal/Entidade promotora: Vídeo transmitido pelo canal da Undime- RN, mas tendo como instituição promotora o Fórum estadual de educação RN

Transmitido ao vivo em: 09 de jun. de 2020

Link de acesso: https://youtu.be/Mq9gSkHaAv4

Título: Dilemas e desafios das/dos profissionais da educação infantil em tempos de pandemia Participantes: Taís Mendes, Marcia Aparecida Baldini, Carlos Sanches, Lucineia Maria Lazaretti, Soeli Pereira

Canal/Entidade promotora: FEIPAR- Fórum de educação infantil do Paraná

Transmitido ao vivo em: 09 de jun. de 2020

Link de acesso: https://youtu.be/tQ6R-JZlgIQ

Título: Racismo - A Educação Infantil precisa falar sobre isso

Participantes: Carina Costa, Rainara Maia e Kilvia Lopes

Canal/Entidade promotora: Canal do Fórum de Educação Infantil do Ceará

Transmitido ao vivo em: 10 de jun. de 2020

Link de acesso: https://youtu.be/5BRIsb2MYdc

Título: 4a Hora da Brincada: Educação infantil em tempos de pandemia (desafios e propostas)

Participantes: Emilia Cipriano, Monika Silva, Natália Tazinazzo Sandra Toquetão, Telma Tsuda, Fernanda Liberali e Crianças

Canal/Entidade promotora: Canal Projeto Brincadas

Transmitido ao vivo em: 12 de junho de 2020

Link de acesso: https://youtu.be/mPlp8ErAPD0

Recebido em: 15 de junho de 2020.

Versão corrigida recebida em: 01 de dezembro de 2020.

Aceito em: 01 de dezembro de 2020.

Publicado online em: 23 de julho de 2021 .

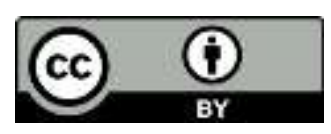

Olhar de professor, Ponta Grossa, v. 24, p. I-18, e-16055.075, 202I.

Disponível em <https://revistas2.uepg.br/index.php/olhardeprofessor> 